\title{
Airway surface liquid homeostasis in cystic fibrosis: pathophysiology and therapeutic targets
}

\author{
Iram J Haq, ${ }^{1,2}$ Michael A Gray, ${ }^{3}$ James P Garnett, ${ }^{1}$ Christopher Ward, ${ }^{1,3}$ \\ Malcolm Brodlie ${ }^{1,2}$
}

${ }^{1}$ Institute of Cellular Medicine, Newcastle University, Newcastle upon Tyne, UK ${ }^{2}$ Department of Paediatric Respiratory Medicine, Great North Children's Hospital, Newcastle upon Tyne, UK ${ }^{3}$ Institute for Cell and Molecular Bioscience, Newcastle University, Newcastle upon Tyne, UK

Correspondence to Dr Malcolm Brodlie, Great North Children's Hospital, Old Children's Outpatient Department, Queen Victoria Road, Newcastle upon Tyne NE1 4LP, UK; malcolm. brodlie@newcastle.ac.uk

Received 18 July 2015 Revised 24 November 2015 Accepted 1 December 2015 Published Online First 30 December 2015

\section{ABSTRACT}

Cystic fibrosis (CF) is a life-limiting disease characterised by recurrent respiratory infections, inflammation and lung damage. The volume and composition of the airway surface liquid (ASL) are important in maintaining ciliary function, mucociliary clearance and antimicrobial properties of the airway. In CF, these homeostatic mechanisms are impaired, leading to a dehydrated and acidic ASL. ASL volume depletion in CF is secondary to defective anion transport by the abnormal cystic fibrosis transmembrane conductance regulator protein (CFTR). Abnormal CFTR mediated bicarbonate transport creates an unfavourable, acidic environment, which impairs antimicrobial function and alters mucus properties and clearance. These disease mechanisms create a disordered airway milieu, consisting of thick mucopurulent secretions and chronic bacterial infection. In addition to CFTR, there are additional ion channels and transporters in the apical airway epithelium that play a role in maintaining ASL homeostasis. These include the epithelial sodium channel (ENaC), the solute carrier $26 \mathrm{~A}$ (SLC26A) family of anion exchangers, and calciumactivated chloride channels. In this review we discuss how the ASL is abnormal in CF and how targeting these alternative channels and transporters could provide an attractive therapeutic strategy to correct the underlying ASL abnormalities evident in CF.

\section{AIRWAY SURFACE LIQUID}

The airway surface liquid (ASL) is a thin layer of fluid covering the luminal (apical) surface of the airway epithelium and plays a key role in airway homeostasis. The volume, $\mathrm{pH}$, ionic and nutrient content of the ASL are all important in regulating antimicrobial activity, ciliary function and mucociliary transport (figure 1). Antimicrobial factors found within the ASL are involved in innate and adaptive host defence mechanisms that protect the airway from inhaled pathogens.

Alterations in ASL composition and disruption of this tightly regulated environment have been linked to the pathogenesis of cystic fibrosis (CF) lung disease. Knowledge of this area has progressed rapidly of late and the focus of this review is to highlight how factors involved in maintaining ASL homeostasis could be potential targets for novel therapeutic strategies in people with CF.

The ASL consists of two distinct layers (figure 1). The mucus layer contains large gel-forming mucins which trap inhaled particles for removal from the lung by mucociliary clearance. Beneath this is the periciliary layer (PCL), which lies adjacent to the airway epithelial cells and surrounds the cilia. High concentrations of large membrane-bound mucins within the PCL are tethered to the cilia and surface of apical epithelial cells. ${ }^{1}$ They form a tight macromolecular mesh, preventing penetration of mucus and inhaled particles into the PCL. The greater osmotic modulus generated by these membrane mucins allows relative preservation of PCL volume in a healthy hydrated airway. ${ }^{1}$

This recent 'gel-on brush' ASL model has enabled greater understanding of how disruption of this tightly regulated system can alter mucus clearance and contribute towards pathogenesis in airways diseases. ${ }^{1}$

\section{THE ASL IS DEHYDRATED IN CF}

In $\mathrm{CF}$, dysfunctional $\mathrm{CF}$ transmembrane conductance regulator (CFTR) protein leads to defective apical chloride and bicarbonate transport into the airway lumen. Subsequent sodium and water compartmental shifts, in addition to this defective anion transport, result in a dehydrated airway surface and the production of viscous, acidic, muco-purulent secretions that are difficult to clear.

In a dehydrated airway environment, water preferentially moves out of the ASL mucus layer, increasing its concentration and osmotic modulus. Eventually, in severely dehydrated CF airways, a critical threshold is reached whereby water also exits the PCL. The mucus layer subsequently compresses the PCL, affecting ciliary activity and therefore mucociliary transport. ${ }^{1}$ In $\mathrm{CF}$, this leads to mucus stasis, airway infection, inflammation, and progressive lung disease (figure 1).

\section{THE ASL PH IS REDUCED IN CF}

Defective CFTR-mediated bicarbonate transport leads to an acidic ASL, which creates an unfavourable environment for the function of many antimicrobial factors. A reduction in ASL $\mathrm{pH}$ in the CF pig model has been shown to decrease antimicrobial activity, which was restored by directly increasing $\mathrm{pH}^{2}{ }^{2}$ Bicarbonate is also required for maintaining normal mucus properties and homeostasis. Its depletion results in dense, impenetrable mucus that remains tethered to the epithelium, thus impairing mucociliary transport. ${ }^{3}$

\section{THERAPEUTIC TARGETS FOR ASL REGULATION IN CF}

The underlying genetic defect in CF is well defined, and recent therapeutic strategies have been directed towards correcting the function of mutant CFTR protein. ${ }^{4}$ For example, the CFTR potentiator, ivacaftor, has proved clinically effective in the $5 \%$ of people with the Gly551Asp-CFTR gating mutation. ${ }^{5}$ In 2015 the results of phase III trials of 

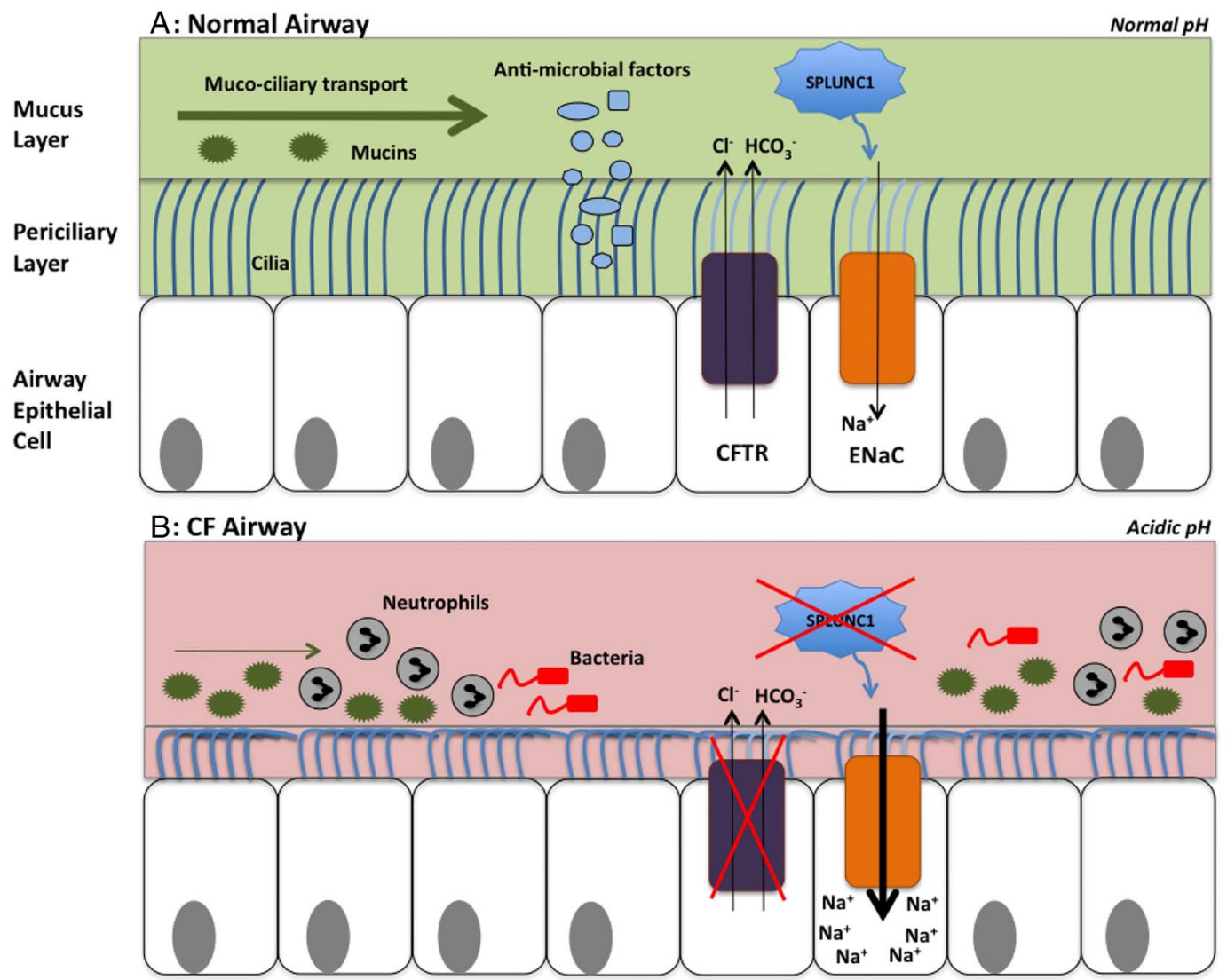

C: Alternative approaches for CF therapy

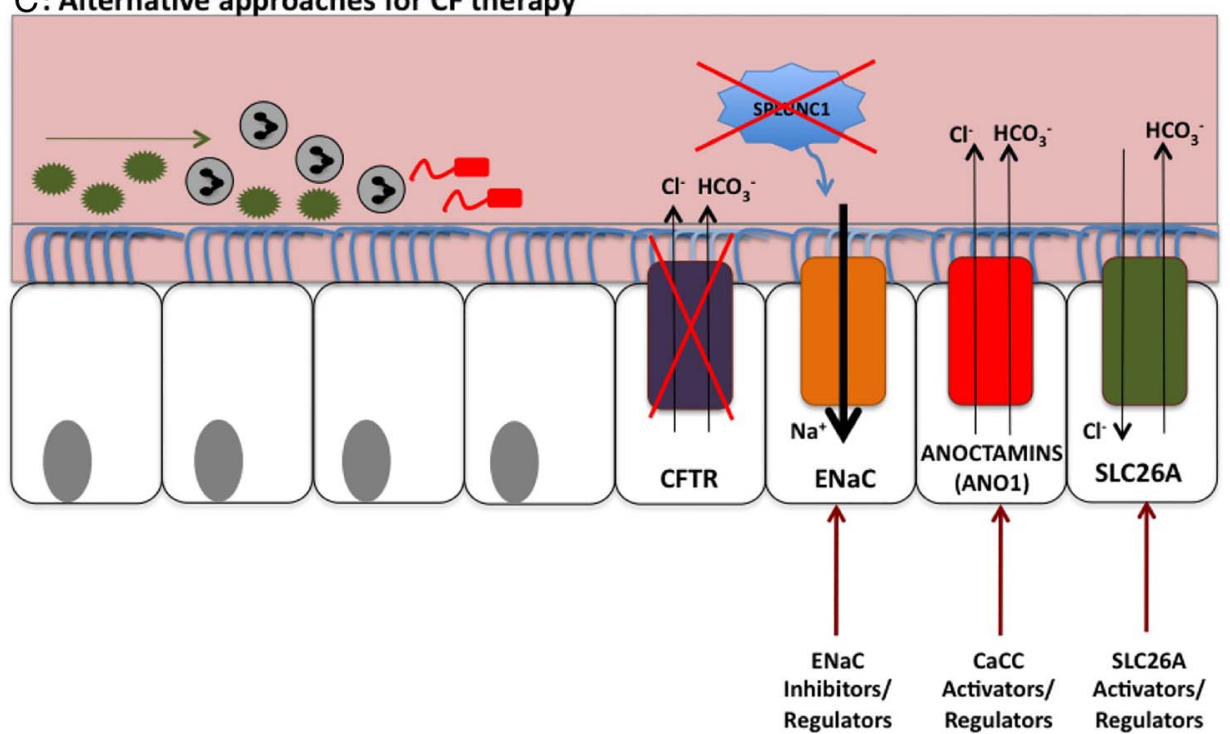

Figure 1 The airway surface liquid (ASL) in cystic fibrosis (CF) and potential targets for therapies. (A) The normal airway: the ASL consists of a mucus layer containing large gel-forming mucins that trap inhaled particles for removal by mucociliary clearance. High concentrations of osmotically active tethered mucins within the periciliary layer $(\mathrm{PCL})$ form a tight macromolecular mesh and preserve $\mathrm{PCL}$ volume. The $\mathrm{CF}$ transmembrane conductance regulator protein (CFTR) transports chloride and bicarbonate into the airway. Sodium is absorbed via the epithelial sodium channel $(\mathrm{ENaC})$, contributing to ASL hydration and mucociliary transport. SPLUNC1 is found in abundance within the ASL and regulates ASL volume by inhibiting ENaC. The ASL also contains antimicrobial factors, which play a key role in innate host defence. (B) The CF airway: defective chloride and bicarbonate transport by abnormal CFTR leads to a dehydrated and acidic ASL. SPLUNC1 fails to regulate ENaC, leading to sodium hyper-absorption and further ASL volume depletion. The acidic environment reduces antimicrobial function and increases mucus viscosity. These mechanisms result in the production of thick, mucopurulent secretions and bacterial colonisation. In this dehydrated state, water preferentially moves out of the mucus layer, increasing its concentration. The PCL volume is initially preserved, but eventually reaches a critical threshold at which water also exits the PCL. The mucus layer subsequently compresses the PCL, compromising ciliary activity and mucociliary clearance. (C) Alternative approaches for CF therapy: targeting other apical channels and transporters may be potential alternative avenues for CF therapy. ENaC inhibition or regulation could help to preserve ASL volume. Regulation or activation of alternative apical chloride channels, including the anion exchanger solute carrier $26 \mathrm{~A}$ (SLC26A) family, and the calcium-activated chloride channel, anoctamin 1, could correct defective apical chloride transport and therefore the ASL abnormalities evident in CF lung disease. 
the combination of ivacaftor and the corrector lumacaftor in patients homozygous for the most frequent CF-causing mutation Phe508del were published, but showed less clinically significant benefits. ${ }^{6}$ The combination drug received US Food and Drug Administration approval in 2015 for treatment in patients who are Phe508del homozygotes. However, this approach will not benefit all patients; around 50\% are not Phe508del homozygotes, and alternative strategies to correct the underlying fluid and $\mathrm{pH}$ imbalance in the ASL are required.

Stimulation of other channels and transporters involved in apical ion transport may compensate for the abnormalities in ion transport, solute trafficking, $\mathrm{pH}$ and ASL volume that are seen with the absence of functional CFTR. These 'by-pass therapies' could provide complementary or even alternative therapies in CF that would benefit all patients.

\section{ENAC}

The epithelial sodium channel, $\mathrm{ENaC}$, plays a key role in apical sodium absorption and is important in regulating ASL hydration and mucociliary transport. $\mathrm{ENaC}$ activity is upregulated in $\mathrm{CF}$, which leads to sodium hyper-absorption and contributes to ASL dehydration (figure 1)..$^{8}$

The precise underlying mechanisms for $\mathrm{ENaC}$ upregulation in $\mathrm{CF}$ are not fully understood but knowledge in this area is increasing. CFTR dysfunction is thought to be linked to an increase in $\mathrm{ENaC}$ activity, however this has not been demonstrated consistently in all experimental models and their relationship remains in question. ${ }^{9}$

Furthermore, ENaC activation occurs through a number of other mechanisms, including the cleavage of its subunits by endogenous serine proteases. ${ }^{10}$ One such example, neutrophil elastase, is abundant in inflamed CF airways and in other diseases including COPD, which may be contributory to $\mathrm{ENaC}$ activation. ${ }^{11} 12$

Given the hyperactivity of $\mathrm{ENaC}$ in $\mathrm{CF}$, inhibitory agents have been investigated as potential therapies. In particular, amiloride has previously failed to show sustained clinical improvements due to low potency and rapid airway absorption. ${ }^{13}$ Other potential agents, including derivatives of amiloride, have since been developed and undergone phase I and II clinical trials. However, challenges have been faced with developing new compounds that provide adequate lung delivery without unwanted pulmonary and systemic side effects, and the development of novel agents is still underway.

The secreted protein, short palate lung and nasal epithelial clone 1 (SPLUNC1) is abundant within the ASL. In normal airways SPLUNC1 regulates ASL volume by binding to and preventing the cleavage of $\mathrm{ENaC}$, thereby inhibiting its action. ${ }^{10}$ Importantly, SPLUNC1 becomes ineffective at inhibiting ENaC when the ASL $\mathrm{pH}$ is reduced. In the acidic, dehydrated CF airway, normal $\mathrm{ENaC}$ regulation is disrupted, resulting in sodium hyper-absorption and further depletion of ASL volume. ${ }^{14}$ Protease inhibitors and $\mathrm{pH}$-insensitive derivatives of SPLUNC1 could prove beneficial in regulating $\mathrm{ENaC}$ activity, but further studies of these targets in human models are required.

$\mathrm{ENaC}$ regulation rather than inhibition may be required to maintain normal ASL homeostasis. A large-scale siRNA screen was performed to identify genes involved in the mechanistic processes for $\mathrm{ENaC}$ regulation. This was coupled with live cell microscopy in human airway epithelial cells to assess $\mathrm{ENaC}$ function. ${ }^{15}$ Over 1500 activators and inhibitors for $\mathrm{ENaC}$ were identified and further detailed investigation highlighted a particular protein, $\mathrm{DGK}$, as a potential target. $\mathrm{DGK}_{\mathrm{l}}$ is required to maintain $\mathrm{ENaC}$ activity, and its inhibition reduced $\mathrm{ENaC}$ activity in CF primary airway epithelial cells. Furthermore, $\mathrm{ENaC}$ activity and airway fluid absorption were normalised, highlighting its potential role as a novel CF therapy. ${ }^{15}$

\section{ALTERNATIVE CHLORIDE CHANNELS AND TRANSPORTERS}

Although $\mathrm{ENaC}$ regulation will benefit sodium hyper-absorption, the correction of dysfunctional chloride and bicarbonate transport will require additional strategies potentially involving the modification of other alternative channels (figure 1). ${ }^{16}$

One such approach is to target calcium activated chloride channels $(\mathrm{CaCC})$. These are widely expressed in a range of tissue types and are diverse in their functions. Anoctamin 1 (Ano1) is a member of the anoctamin transmembrane protein family and is an essential component of the CaCC. ${ }^{17}$ Amongst many other tissues, it is expressed in the airway epithelium and is active in CF airways. Ano1 knockout mice develop a CF-like phenotype, highlighting its importance in chloride transport and potential role for therapy. ${ }^{18}$ Upregulation of Ano1 may be successful in compensating for CFTR dysfunction in CF.

Challenges have been faced with developing agents that utilise $\mathrm{CaCC}$ pathways, initially due to poor airway potency. An alternative longer-acting $\mathrm{P}_{2} \mathrm{Y}_{2}$ receptor agonist, denufosol, did not show any significant improvements in lung function in phase III trials. Although this area holds promise, further investigation is required to establish potential therapies.

Another approach would be to target the solute carriers (SLC), a family of plasma membrane anion exchangers and channels that are widely expressed throughout the body. In particular, the SLC26A members of this family are involved in chloride/bicarbonate exchange in secretory and airway epithelia and have been found to have regulatory interactions with CFTR. ${ }^{19} 20$ A greater understanding of these interactions together with further investigation of their significance in the CF airway could potentially provide some promising potential targets for therapy.

\section{CONCLUSIONS AND FUTURE DIRECTIONS}

The ASL plays a key role in mucus clearance and lung defence. In addition to CFTR, additional apical channels and transporters are involved in ion transport, and could be potential strategies for CF therapy.

Challenges may be faced given the wide expression of many of these proteins, the subsequent risk of potential unwanted systemic effects and the additional challenge of achieving adequate drug potency within the airways. Furthermore, the dynamic assessment of the ASL in vivo is challenging, as is the functional assessment of its response to future druggable targets.

Further development of representative human models would be necessary to enable the investigation of these promising avenues for future CF management. Targeting a combination of these bypass channels could successfully correct the ASL abnormalities seen in CF, providing benefit to all patients regardless of their mutation.

Contributors All authors developed a plan for the article, IJH and MB wrote the first draft that all authors commented on, IJH and MB then completed the final version.

Funding MAG, CW and MB: Strategic Research Centre Grant (SRC003), Cystic Fibrosis Trust. MB: Medical Research Council Clinician Scientist Fellowship (MR/M008797/1). JPG: Newcastle University Wellcome Trust Institutional Strategic Support Fund.

Competing interests None declared.

Provenance and peer review Not commissioned; externally peer reviewed. 


\section{REFERENCES}

1 Button B, Cai L-H, Ehre C, et al. A periciliary brush promotes the lung health by separating the mucus layer from airway epithelia. Science 2012;337:937-41.

2 Pezzulo AA, Tang XX, Hoegger MJ, et al. Reduced airway surface pH impairs bacterial killing in the porcine cystic fibrosis lung. Nature 2012;487:109-13.

3 Gustafsson JK, Ermund A, Ambort D, et al. Bicarbonate and functional CFTR channel are required for proper mucin secretion and link cystic fibrosis with its mucus phenotype. J Exp Med 2012;209:1263-72.

4 Brodlie M, Haq IJ, Roberts K, et al. Targeted therapies to improve CFTR function in cystic fibrosis. Genome Med 2015;7:101.

5 Ramsey BW, Davies J, McElvaney NG, et al. A CFTR potentiator in patients with cystic fibrosis and the G551D mutation. N Eng/ J Med 2011;365:1663-72.

6 Wainwright CE, Elborn JS, Ramsey BW, et al. Lumacaftor-ivacaftor in patients with cystic fibrosis homozygous for Phe508del CFTR. N Engl J Med 2015;373:220-31.

7 Stutts MJ, Canessa CM, Olsen JC, et al. CFTR as a CAMP-dependent regulator of sodium channels. Science 1995;269:847-50.

8 Donaldson SH, Boucher RC. Sodium channels and cystic fibrosis. Chest 2007;132:1631-6.

9 Collawn JF, Lazrak A, Bebok Z, et al. The CFTR and ENaC debate: how important is ENaC in CF lung disease? Am J Physiol Lung Cell Mol Physiol 2012;302:L1141-6.

10 Garcia-Caballero A, Rasmussen JE, Gaillard E, et al. SPLUNC1 regulates airway surface liquid volume by protecting $\mathrm{ENaC}$ from proteolytic cleavage. Proc Natl Acad Sci USA 2009;106:11412-17.
11 Gaillard EA, Kota P, Gentzsch M, et al. Regulation of the epithelial Na+ channel and airway surface liquid volume by serine proteases. Pflugers Arch 2010;460:1-17.

12 Weldon S, McNally P, McElvaney NG, et al. Decreased levels of secretory leucoprotease inhibitor in the Pseudomonas-infected cystic fibrosis lung are due to neutrophil elastase degradation. J Immunol 2009;183:8148-56.

13 Knowles MR, Church NL, Waltner WE, et al. A pilot study of aerosolized amiloride for the treatment of lung disease in cystic fibrosis. $N$ Engl J Med 1990;322:1189-94.

14 Garland AL, Walton WG, Coakley RD, et al. Molecular basis for pH-dependent mucosal dehydration in cystic fibrosis airways. Proc Natl Acad Sci USA 2013;110:15973-8.

15 Almaça J, Faria $D$, Sousa $M$, et al. High-content siRNA screen reveals global ENaC regulators and potential cystic fibrosis therapy targets. Cell 2013;154:1390-400.

16 Mall MA, Galietta LJ. Targeting ion channels in cystic fibrosis. J Cyst Fibros 2015; 14:561-70.

17 Jang Y, Oh U. Anoctamin 1 in secretory epithelia. Cell Calcium 2014;55:355-61.

18 Rock JR, O'Neal WK, Gabriel SE, et al. Transmembrane protein 16A (TMEM16A) is a Ca2+-regulated $\mathrm{Cl}$ - secretory channel in mouse airways. J Biol Chem 2009;284:14875-80.

19 Alper SL, Sharma AK. The SLC26 gene family of anion transporters and channels. Mol Aspects Med 2013;34:494-515.

20 Ko SB, Shcheynikov N, Choi JY, et al. A molecular mechanism for aberrant CFTR-dependent HCO3-transport in cystic fibrosis. EMBO J 2002;21:5662-72. 